\title{
¿Por qué estudiar letras modernas y lenguas extranjeras?
}

\author{
Édgar Alejandro AgUILAR MAYORGA \\ Universidad Nacional Autónoma de México
}

\begin{abstract}
El siguiente trabajo aborda, de manera breve, la importancia de aprender lenguas y literaturas extranjeras, partiendo de la experiencia personal en relación con las letras portuguesas, mediante la reflexión acerca de la lengua y sus implicaciones culturales, entre ellas, el conocimiento del otro y el reconocimiento de identidades y realidades lingüísticas diversas que dan cuenta de lo humano, tanto a nivel social como individual. Se esboza, además, la importancia de la reciente incorporación de las letras portuguesas en la Facultad de Filosofía y Letras de la UNAM como respuesta a la necesidad misma de aprender y enseñar lengua y literaturas portuguesas en México.
\end{abstract}

PALABRAS CLAVE: lenguas-culturas, letras portuguesas, enseñanza-aprendizaje, lenguas extranjeras, literaturas extranjeras.

The following work discusses, briefly, the importance of learning foreign languages and literature, starting from the personal experience in relation to the Portuguese letters. In this essay subjects like language and its cultural implications; the knowledge of the other and the recognition of diverse identities and linguistic realities that account for the existence human beings in individual and social levels, are considered. It also outlines the importance of the recent addition of Portuguese letters in the Faculty of Philosophy and Letters of the UNAM in response to the need to learn and teach Portuguese language and literature in Mexico.

KEY WORDS: languages-cultures, Portuguese letters, teaching-learning, foreign languages, foreign literature.

Comenzaré refiriendo y compartiendo una experiencia muy personal con respecto a mi interés por la lengua portuguesa.

La lengua portuguesa llegó a mis ojos y oídos por primera vez hace casi doce años cuando comenzaba a estudiar en la preparatoria, reforzando mi gusto por la lectura e introduciendo, también dentro de mis disfrutes, una música cuyos sonidos y voces se irían adentrando cada vez más en la construcción de mi identidad adolescente. La caverna de José Saramago y la música popular brasileña fueron, hasta cierto punto, los principales motivos que me condujeron hacia Portugal y Brasil al mismo tiempo; un 
conjunto de ventanas que aparecieron ante mí esperando a ser abiertas, descubiertas, a través de una musicalidad que he considerado siempre presente en esta lengua. Mis pasos en abrir y descubrir dichas ventanas fueron posibles gracias al acceso a medios de comunicación como el internet, donde la radio y la lectura de diversos tipos de texto hacían posibles las voces, su musicalidad, las palabras, en fin, el aprendizaje de una lengua que, en un principio, me parecía muy semejante al español y que, al final, terminaría por constituirse más compleja. Escuchaba el portugués e imitaba, leía el portugués e imitaba, siempre en el afán de comprender y producir un nuevo código de una nueva realidad para mí; realidad que habría de dividirse después habiendo descubierto las semejanzas y las diferencias entre el portugués europeo y el portugués brasileño, adoptándome, de manera lúdica, a una u otra variante o a una u otra realidad, pues mis nuevos gustos dependían, y dependen todavía, de ambas. Ya en el momento decisivo de estudiar una carrera universitaria y habiéndome familiarizado con el portugués, me inclinaba por enseñar lenguas extranjeras, quizá por esta experiencia de autoaprendizaje que había emprendido; sin embargo, en aquel entonces no existía una carrera que me permitiera conocer la literatura y la lengua portuguesas de manera formal a nivel universitario, por lo que opté por ingresar a la licenciatura en Letras Hispánicas, la cual amplió mi visión de la literatura y la lingüística, satisfaciendo mis expectativas profesionales. No obstante, mi deseo de adentrarme en la literatura y la lengua portuguesas no había quedado satisfecho hasta que en 2010 apareció, finalmente, la oportunidad de realizarlo, pese a todo obstáculo.

De la experiencia anterior, y desde mi posición de estudiante y profesor, considero que el aprendizaje de una lengua extranjera es motivado por intereses que pueden ir desde lo literario y lo musical hasta lo económico y lo mercadotécnico; intereses determinados por un gusto personal, o bien, por una necesidad profesional. Si bien dichos intereses pueden ser múltiples, el aprendizaje de una lengua extranjera siempre nos conduce al aprendizaje de una cultura extranjera también, pues, como bien se defiende en las posturas de la antropología lingüística, toda lengua es determinada por una cultura, entendida ésta como una realidad compleja que se compone de un aparato ideológico sociocultural e histórico propio, el cual termina por delimitar una realidad lingüística. Así pues, hemos de identificar el aprendizaje de lenguas extranjeras como el tránsito entre dos realidades, la propia, o materna, y la otra, o extranjera; tránsito en que interactúan dichas realidades, pues a partir de lo propio es que nos es posible definir lo otro y a partir de lo otro podemos definir lo propio, en un tipo de intercambio que nos lleva al conocimiento del otro, pero también, al autoconocimiento, donde yace quizá la mayor importancia de aprender una lengua extranjera, pues dichos tipos de conocimiento no tienen más que como producto final la ampliación o extensión de un panorama único o de una realidad única dividida tan sólo por la misma diversidad lingüística, como si cada lengua, con todo y su trasfondo cultural, fuera solamente un fragmento de un suceso único, lo humano.

De esta manera, hablar de la lengua portuguesa es hablar de ocho realidades (Angola, Brasil, Cabo Verde, Guiné Bissau, Mozambique, Portugal, San Tomé y Príncipe, 
Timor Este), pues si bien la lengua es común a estos ocho países, no dejan de cumplir un papel fundamental los hábitos, las costumbres, los hechos y los objetos, puesto que forman parte de una realidad que se denomina solamente a través de la lengua y una lengua que se conforma mediante su relación con la realidad. El aprendizaje de una lengua extranjera es, pues, una especie de llave maestra que nos da acceso directo, en el caso del portugués, a estas ocho realidades desde los más diversos puntos de vista, dependiendo siempre de los intereses de cada aprendiente. Así, aprender una lengua extranjera se convierte en una tarea de exploración, pues, como ya se mencionó anteriormente, durante este tránsito entre culturas o realidades se van hallando y descubriendo elementos sociales, históricos y culturales propios de cada lengua o de cada variante de lengua. El aprendizaje de una lengua extranjera actualmente debe constituirse como un reflejo del constante intercambio económico, social, cultural, en fin, histórico, logrado gracias a los avances tecnológicos que tienden los puentes para el alcance de contactos más directos, más estrechos, entre la mayoría de las sociedades. Actualmente, no es de sorprenderse encontrar en México personas que hablan portugués, italiano, francés, griego, chino, hindi, bengalí, coreano; personas que se integran a nuestra realidad y a nuestra lengua, estrechando y celebrando con México lazos económicos o académicos, permitiéndonos ver que el intercambio se ha transformado en parte fundamental de nuestra realidad.

Cuando nos referimos al estudio del portugués en México, hablamos de medios de comunicación brasileños y portugueses que intervienen en colaboración con los medios de comunicación nacionales; hablamos de las intermitencias de la música brasileña, portuguesa, angolana y mozambicana en nuestra radio; hablamos de investigación en diferentes áreas de conocimiento que van de lo arquitectónico, lo histórico, lo antropológico, lo literario y lo lingüístico, justo aquí en la Universidad. Lo anterior no demuestra más que un interés de los mexicanos hacia las ocho realidades ya mencionadas anteriormente, pero, al mismo tiempo, la inserción o la intrusión de éstas gracias al intercambio al que ya me he referido. En México, hay farmacéuticas brasileñas, y en Brasil, farmacéuticas mexicanas. En Portugal, hay cuarenta empresas mexicanas y en México igual número de empresas portuguesas.* El portugués, en este punto, adquiere una relevancia social, cultural y económica que poco a poco se ha venido ampliando y confirmando con la aparición no sólo de empresas, sino de cátedras, institutos de lengua y centros de estudios: en 1987, la cátedra Guimarães Rosa; en 2004, la cátedra José Saramago; en 2002, el Instituto Camões; en 2010, la licenciatura en Lengua y Literaturas Modernas Portuguesas. Apariciones que confirman el interés de México por el mundo lusófono, por estas ocho realidades que nos han llegado para ser exploradas a través de una lengua hermana, geográfica e históricamente.

Ya en el caso específico de la Facultad de Filosofía y Letras, la licenciatura en Letras Portuguesas constituye no solamente un logro — pues finalmente los estudiosos de la

\footnotetext{
* Datos cedidos por la Embajada de Portugal en México a través del Instituto Camões.
} 


\section{$234 \square$ ¿POR QUÉ ESTUDIAR LETRAS MODERNAS Y LENGUAS EXTRANJERAS?}

lengua y las culturas portuguesas anteriores habrán de conseguir la unión de un rompecabezas, cuyas piezas vagaban esparcidas en un espectro que parecía concentrar, de forma aislada, el mundo de la lusofonía en nuestro país-, sino también un punto de partida para quienes nos profesionalizamos de una manera más plena en las ocho realidades de la lengua portuguesa, teniendo la responsabilidad de enseñar mejor, de traducir mejor, de investigar mejor, las lenguas y las literaturas portuguesas, o lo portugués en al ámbito humanístico, algo que, sin duda alguna, se vislumbra ya como significativo para el portugués en México. Estudiar letras portuguesas es, pues, aventurarse a transmitir nuevas perspectivas sobre el mundo lusófono a través de la enseñanza y la investigación; implica traer nuevas literaturas a nuestro país, a través de la traducción de nuevos autores, de autores desconocidos del pasado y del presente, ampliando el trabajo hasta ahora emprendido por investigadores y profesores no sólo de nuestra Universidad, sino también de otras instancias. Estudiar letras portuguesas es apostar por el fortalecimiento de los vínculos comerciales y socioculturales entre México y los ocho países de lengua oficial portuguesa.

¿Por qué estudiar letras modernas y lenguas extranjeras? Porque en su acercamiento hemos de ser capaces de transmitir y difundir culturas extranjeras que emergen en nuestra realidad (en nuestra cultura), comprendiéndolas a través de sus lenguas en su complejo histórico, social, ideológico y económico, haciendo que otros las comprendan, las adopten como propias también y sepan "con-vivirlas".

\section{Obras consultadas}

BoAs, Franz. 1940. Race, Language, and Culture. Nueva York: Columbia University.

CARCEDO, Alberto. 2000. "La lengua como manifestación de otredad cultural (o convergencia cultural)". Espéculo. Revista de estudios literarios. Universidad Complutense de Madrid. 9 de noviembre de 2011. <http//www.ucrn.es/info/ especulo/ele>.

MaLINOWSKI, Bronislaw. 1981. Una teoría científica de la cultura. Barcelona: Edhasa. 